\title{
Two-Dimensional Dilaton Gravity
}

\author{
Marco Cavaglià \\ Max-Planck-Institut für Gravitationsphysik, Albert-Einstein-Institut, \\ Schlaatzweg 1, D-14473 Potsdam, Germany ${ }^{1}$
}

\begin{abstract}
I briefly summarize recent results on classical and quantum dilaton gravity in $1+1$ dimensions.
\end{abstract}

\section{INTRODUCTION}

In the last few years a great deal of activity has been devoted to the investigation of lower-dimensional gravity [1]. The interest on dimensionally reduced theories of gravity relies essentially on their relation to string theory, higher-dimensional gravity, black hole physics, and gravitational collapse. In this talk I will focus attention on the simplest, non-trivial, lower-dimensional theory of gravity: $1+1$ (pure) dilaton gravity [2].

Dilaton gravity is described by the action

$$
S\left[\phi, g_{\mu \nu}\right]=\int d^{2} x \sqrt{-g}[\phi R(g)+V(\phi)],
$$

where $\phi$ is the dilaton field, $V(\phi)$ is the dilatonic potential, and $R$ is the twodimensional Ricci scalar. In Eq. (1) we have used a Weyl-rescaling of the metric to eliminate the kinetic term of the dilaton field. Equation (1) describes a family of models whose elements are identified by the dilatonic potential. For instance, $V(\phi)=$ constant identifies the matterless sector of the Callan-Giddings-HarveyStrominger model (CGHS) [3], $V(\phi)=\phi$ identifies the Jackiw-Teitelboim model, and $V(\phi)=2 / \sqrt{\phi}$ describes the two-dimensional sector of the four-dimensional spherically-symmetric Einstein gravity after having integrated on the two-sphere with area $4 \pi \phi$.

Dilaton gravity is an interesting example of Completely Integrable Model, i.e. a model that can be expressed in terms of free fields by a canonical transformation. Completely integrable models play an important role from the point of view of the quantum theory because they can be quantized exactly (in the free-field representation). This property allows the discussion of quantization subtleties and

1) E-mail: cavaglia@aei-potsdam.mpg.de, web page: http://www.aei-potsdam.mpg.de/ ${ }^{\sim}$ cavaglia 
non-perturbative quantum effects. (For the CGHS model see for instance Refs. [4].) Since dilaton gravity can be used to describe black holes and/or gravitational collapse (in the case of coupling with matter), the quantization program is worth exploring.

A direct consequence of the complete integrability of dilaton gravity is that both the metric and the dilaton can be expressed in terms of a D'Alembert field and of a local integral of motion independent of the coordinates [5]. So, using the gauge in which the free field is one of the coordinates, one finds that all solutions depend on a single coordinate. This property constitutes a generalization of the classical Birkhoff Theorem. (For spherically-symmetric Einstein gravity, i.e. $V(\phi)=$ $2 / \sqrt{\phi}$, the "local integral of motion independent of the coordinates" is just the Schwarzschild mass and the dependence of both the metric and the dilaton from a single D'Alembert field means that the four-dimensional line element can be written in a form depending on the radial coordinate only.)

So dilaton gravity can be quantized using two alternative, a priori non-equivalent, approaches. In the first one the theory is quantized by first reducing it to a $0+1$ dynamical system, i.e. using first the classical Birkhoff theorem and then the quantization algorithm. Conversely, in the second approach the theory is quantized in the full $1+1$ sector and the $0+1$ dimensional nature of the system must be recovered a posteriori (Quantum Birkhoff Theorem) [6]:

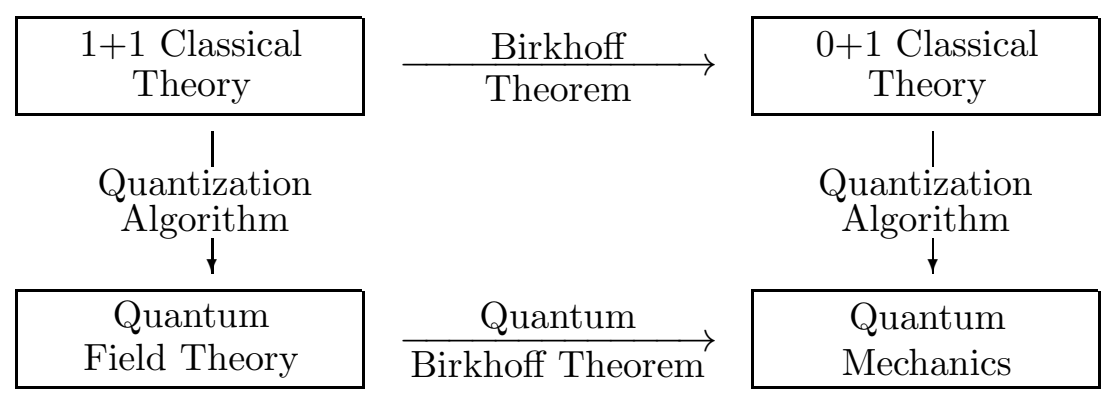

Furthermore, because of the gauge nature of the theory, the quantization of the system can be implemented according to two different procedures: the Dirac method - quantization of the constraints followed by gauge fixing - and the reduced canonical method - classical gauge fixing followed by quantization in the reduced space. Usually, the two methods do not lead to identical results.

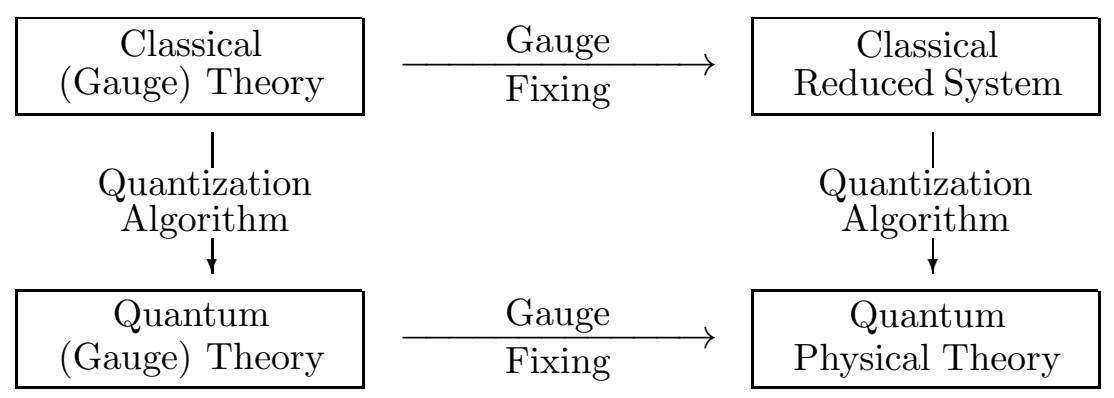


Here I will show that both diagrams close and the different approaches are equivalent. At the end of the talk I will briefly discuss why these conclusions fail in the case of dilaton gravity coupled to scalar matter.

\section{II $0+1$ QUANTIZATION}

In the $0+1$ approach the proof of the equivalence of Dirac and reduced methods is straightforward because we are able to pass, via a canonical transformation, to a maximal set of gauge-invariant canonical variables.

Using obvious notations the $0+1$ action reads

$$
S_{0+1}=\int d t\left[\dot{q}_{i} p_{i}-\mu H\right], \quad i=1,2,
$$

where $\mu$ is a Lagrange multiplier enforcing the constraint $H=0$. Thus in the $0+1$ sector of the theory we can express the field equations as a canonical system in a finite, $2 \times 2$ dimensional, phase space.

Clearly, due to the complete integrability of the model, the equations of motion are analytically integrable and their solution coincides with the finite gauge transformation generated by the (single) constraint $H=0$. So we can find a couple of gauge-invariant independent canonical quantities $\left(M, P_{M}\right)$ and construct the maximal gauge-invariant canonical chart $\left(M, P_{M}, H, T\right)$. Now $T$ can be used to fix the gauge because its transformation properties for the gauge transformation imply that time defined by this variable covers once and only once the symplectic manifold, i.e. time defined by $T$ is a global time. The quantization becomes trivial and both Dirac and reduced approaches lead to the same Hilbert space. The Hilbert space is spanned by the eigenvectors of the (gauge invariant) operator $M$ corresponding to the "mass" of the system introduced in the previous section.

The quantization program illustrated above has been implemented in detail in Refs. [7] for the case of spherically-symmetric Einstein gravity but can be easily generalized to an arbitrary $V(\phi)$. In the case of Refs. [7] one can go further and prove that the Hermitian operator $M$ in the gauge fixed, positive norm, Hilbert space is not self-adjoint, while its square is a self-adjoint operator with positive eigenvalues. This result is due to the fact that the conjugate variable to the "mass" $M$, $P_{M}$, has positive support, analogously to what happens for the radial momentum in ordinary quantum mechanics. It would be interesting to explore whether this conclusion holds for other choices of the dilatonic potential. In any case, what is important for the present discussion is that the mass $M$ - or its square - is the only gauge-invariant observable of the system (apart from the conjugate variable, of course).

\section{1+1 REDUCED QUANTIZATION}

The reduced quantization of the full $1+1$ theory can be implemented using "geometrodynamical-like" canonical variables similar to the canonical variables in- 
troduced by Kuchăr for the canonical description of the Schwarzschild black hole [8]. The new variables are directly related to the spacetime geometry and the relation to the $0+1$ formalism is straightforward.

Let us introduce the ADM parametrization of the metric

$$
g_{\mu \nu}=\rho\left(\begin{array}{cc}
\alpha^{2}-\beta^{2} & \beta \\
\beta & -1
\end{array}\right)
$$

where $\alpha\left(x_{0}, x_{1}\right)$ and $\beta\left(x_{0}, x_{1}\right)$ play the role of the lapse function and of the shift vector respectively, and $\rho\left(x_{0}, x_{1}\right)$ represents the dynamical gravitational degree of freedom. Using Eq. (5), the two-dimensional action in the Hamiltonian form reads

$$
S\left[\phi, g_{\mu \nu}\right]=\int d^{2} x \sqrt{-g}\left[\dot{\phi} \pi_{\phi}+\dot{\rho} \pi_{\rho}-\alpha \mathcal{H}_{0}-\beta \mathcal{H}_{1}\right] .
$$

We can pass to a new canonical chart $\left(M, \pi_{M}, \bar{\phi}, \pi_{\bar{\phi}}\right)$ using the canonical map

$$
\begin{array}{rlrl}
M & =N(\phi)-\frac{\rho^{2} \pi_{\rho}^{2}-\phi^{2}}{\rho}, & \pi_{M} & =\frac{\rho^{2} \pi_{\rho}}{\rho^{2} \pi_{\rho}^{2}-\phi^{2}}, \\
\bar{\phi}=\phi, & \pi_{\bar{\phi}}=\pi_{\phi}-\frac{\rho^{2} \pi_{\rho}}{\rho^{2} \pi_{\rho}^{2}-\phi^{\prime 2}}\left[V(\phi)+2 \pi_{\rho}\left(\frac{\phi^{\prime}}{\rho \pi_{\rho}}\right)^{\prime}\right] .
\end{array}
$$

The canonical quantity $M$ corresponds to the local integral of motion mentioned in Sec. I and can be identified with the mass of the system. In the $0+1$ sector $M$ reduces to the quantity defined in Sec. II. In the new canonical chart the ADM super-Hamiltonian and super-momentum constraints read

$$
\mathcal{H}_{0}=[N(\phi)-M] \pi_{\bar{\phi}} \pi_{M}+[N(\bar{\phi})-M]^{-1} \bar{\phi}^{\prime} M^{\prime}, \quad \mathcal{H}_{1}=-\bar{\phi}^{\prime} \pi_{\bar{\phi}}-M^{\prime} \pi_{M},
$$

where' means differentiation w.r.t. the spatial coordinate $x_{1}$.

The canonical action (6) must be complemented by a boundary term at the spatial infinities. This can be done along the lines of Refs. [8]. The resulting boundary term is of the form

$$
S_{\partial}=-\int d x_{0}\left(M_{+} \alpha_{+}+M_{-} \alpha_{-}\right)
$$

where $M_{ \pm} \equiv M\left(x_{0}, x_{1}= \pm \infty\right)$ and $\alpha_{ \pm}\left(x_{0}\right)$ parametrize the action at infinities.

Now we can solve the constraints and quantize the theory. It is easy to prove that the general solution of Eqs. (9) is given by

$$
\pi_{\bar{\phi}}=0, \quad M^{\prime}=0 .
$$

(Note that $M$ weakly commutes with the constraints, as expected for a local integral of motion.) Thus $M \equiv m\left(x_{0}\right)$ and the effective Hamiltonian is simply given by the boundary term (10). The reduced action reads 


$$
S[m]=\int d \tau\left[\frac{d m}{d \tau} p-m\right],
$$

where $p=\int_{\mathbb{R}} d x_{1} \pi_{M}$ and $\tau\left(x_{0}\right)=\int{ }^{x_{0}} d x_{0}^{\prime}\left(\alpha_{+}+\alpha_{-}\right)$. Now quantization can be carried on as usual. The Schrödinger equation is

$$
i \frac{\partial}{\partial \tau} \psi(m ; \tau)=H_{\mathrm{eff}} \psi(m ; \tau), \quad H_{\mathrm{eff}} \equiv m .
$$

The stationary states are the eigenfunctions of $m$ and the Hilbert space coincides with the Hilbert space obtained in the $0+1$ approach.

\section{1+1 DIRAC QUANTIZATION}

The equivalence between $0+1$ and $1+1$ Dirac methods can be easily proved using the canonical transformation illustrated in the previous section. However, the same result can be obtained through a completely different quantization scheme. Let me sketch the main points.

Since dilaton gravity is completely integrable [5], it seems reasonable to assume the existence of a canonical transformation mapping the original system to a system described by a pair of (constrained) free fields $A_{\alpha}(\alpha=0,1)$ in a flat twodimensional Minkowski background. (In the CGHS case, i.e. constant dilatonic potential, this canonical transformation is explicitly known since long time [4]. A generalization to linear and exponential dilatonic potentials has been recently derived by Cruz and Navarro-Salas, see Ref. [9].) Using the free fields $A_{\alpha}$ the super-Hamiltonian and super-momentum constraints read

$$
\begin{gathered}
\mathcal{H}_{0}=\frac{1}{2} \pi^{\alpha} \pi_{\alpha}+\frac{1}{2} A^{\prime \alpha} A_{\alpha}^{\prime}=0, \\
\mathcal{H}_{1}=-\pi^{\alpha} A_{\alpha}^{\prime}=0
\end{gathered}
$$

where $\pi^{\alpha}$ are the conjugate momenta of $A_{\alpha}$. Now the theory can be quantized in the free field representation. This has been done in detail in Ref. [6] for the CGHS model. (See also Refs. [4].) Since in this case the canonical transformation is known explicitly, the equivalence with the previous approaches can be proved.

In the CGHS case the functional $M$ defined in Sec. I and Sec. III is [6]

$$
M=M_{0}+M_{1}\left(A_{\alpha}, \pi_{\alpha}\right),
$$

where $M_{0}$ is a constant (zero mode). On the field equations we have $M=M_{0}$. Due to positivity conditions that are present in the model the constraints (14-15) can be linearized and the quantization is carried out by use of the standard Gupta-Bleuler method [6].

The quantum reduction of the theory to a $0+1$ dynamical system can be made clear by investigating the matrix elements of the operator $M$. Adopting a normal ordering the matrix elements of $M$ between physical states are 


$$
<\Psi_{2}|M| \Psi_{1}>=<\Psi_{2}\left|M_{0}\right| \Psi_{1}>.
$$

Since $M_{0}$ is a zero mode, it commutes with all the creation and annihilation operators of $A_{\alpha}$. So the vacuum must be labeled by the eigenvalue of $M_{0}$, i.e.

$$
M_{0}|0 ; m>=m| 0 ; m>\text {. }
$$

The existence of infinite vacua, differing by the eigenvalue of the mass, implies that the theory reduces to quantum mechanics. Again, the only gauge invariant operator is the mass (and its conjugate momentum) and the resulting Hilbert space is spanned by the eigenvectors of $M$.

\section{COUPLING TO A MASSLESS SCALAR FIELD}

Let me conclude this talk spending few words on dilaton gravity coupled to a massless scalar field. We have seen that the topological nature of dilaton gravity is a direct consequence of the existence of a functional of the canonical variables which is conserved under time and space translations (the mass $M$ ): the original fields can be expressed in terms of a free field and a local integral of motion instead of two free fields, as one might expect from the counting of the degrees of freedom.

When a scalar field is coupled to the system non-static solutions appear, the Birkhoff theorem is no longer valid, and the topological nature of dilaton gravity is destroyed. This has an important consequence from the canonical point of view. Indeed, we can immediately conclude that no local integrals of motion like $M$ do exist. A provocative interpretation of this result is that the mass of a spherically symmetric black hole coupled to scalar matter cannot be defined at the canonical level! In my opinion this is quite worrying, especially from the quantum point of view. Finally, a related point is that $0+1$ dimensional solutions of dilaton gravity coupled to scalar matter have no horizons - see Refs. [5] and, for the case $V(\phi)=$ $2 / \sqrt{\phi}$, Ref. [10] - or, in other words, "black holes have no scalar hair".

The difficulty in the quantization of dilaton gravity coupled to a massless scalar field is evident in any of the procedures described above. For instance, even though the canonical transformation to free fields described in Sect. IV can be formally implemented, the linearization of the constraints at the basis of the Gupta-Bleuler quantization is no longer possible. Anomalies are present and a consistent quantization requires a modification of the theory [4].

\section{ACKNOWLEDGEMENTS}

I am grateful to the organizers of the conference Particles, Fields $\mathscr{E}$ Gravitation '98 for hospitality and financial support. I am indebted to my friends and collaborators Vittorio de Alfaro and Alexandre T. Filippov for interesting discussions and useful suggestions on various questions connected to the subject of this paper. This work has been supported by a Human Capital and Mobility grant of the European Union, contract no. ERBFMRX-CT96-0012. 


\section{REFERENCES}

1. An updated collection of papers on lower-dimensional gravity can be found at the web page http://www.aei-potsdam.mpg.de/ ${ }^{\sim}$ cavaglia/ldg.html.

2. Recently, dilaton gravity coupled to various matter fields has also been extensively investigated. See for instance: Jackiw, R., in: Procs. of the Second Meeting on Constrained Dynamics and Quantum Gravity, Nucl. Phys. (Proc. Suppl.) 57, 162 (1997); Klösch, T., ibid. p. 326; Strobl, T., ibid. p. 330; Cavaglià, M., Phys. Rev. D 57, 5295 (1998); Phys. Lett. B 413, 287 (1997); Pelzer, H., and Strobl, T., Generalized 2d Dilaton Gravity with Matter Fields, e-Print Archive: gr-qc/9805059; Cavaglià, M., Fatibene, L., and Francaviglia, M., Two-Dimensional Dilaton-Gravity Coupled to Massless Spinors, e-Print Archive: hep-th/9801155, and references therein.

3. Callan, C., Giddings, S., Harvey, J., and Strominger, A., Phys. Rev. D 45, 1005 (1992); Verlinde, H., in: Sixth Marcel Grossmann Meeting on General Relativity, M. Sato and T. Nakamura eds. (World Scientific, Singapore, 1992).

4. Benedict, E., Jackiw, R., and Lee, H.-J., Phys. Rev. D 54, 6213 (1996); Cangemi, D., Jackiw, R., and Zwiebach, B., Ann. Physics (N.Y.) 245, 408 (1995); Kuchař, K.V., Romano, J.D., and Varadarajan, M., Phys. Rev. D 55, 795 (1997).

5. Filippov, A.T., in: Problems in Theoretical Physics, Dubna, JINR, June 1996, p. 113; Mod. Phys. Lett. A 11, 1691 (1996); Int. J. Mod. Phys. A 12, 13 (1997).

6. Cavaglià, M., de Alfaro, V., and Filippov, A.T., Phys. Lett. B 424, 265 (1998), e-Print Archive: hep-th/9802158.

7. Cavaglià, M., de Alfaro, V., and Filippov, A.T., Int. J. Mod. Phys. D 4, 661 (1995); Int. J. Mod. Phys. D 5, 227 (1996).

8. Kuchař, K.V., Phys. Rev. D 50, 3961 (1994); Varadarajan, M., Phys. Rev. D 52, 7080 (1995).

9. Cruz, J., and Navarro-Salas, J., Mod. Phys. Lett. A 12, 2345 (1997).

10. Cavaglià, M., and de Alfaro, V., Int. J. Mod. Phys. D 6, 39 (1997). 